\title{
Impact of Microstrucutre on Dwell Fatigue in Dual-Phase Titanium Alloys
}

\author{
Michelle Harr ${ }^{\mathrm{a}}$, Adam Pilchak ${ }^{\mathrm{b}}$, Samantha Daly ${ }^{\mathrm{c}}$ \\ ${ }^{\mathrm{a}}$ Ph.D. student, Department of Mechanical Engineering, University of Michigan, 2350 Hayward, Ann Arbor, MI 48109, USA \\ ${ }^{\mathrm{b}}$ Senior Materials Research Engineer, Air Force Research Laboratory, Materials and Manufacturing Directorate, 2230 Tenth St., Wright Patterson
} AFB, OH 45433

c Associate Professor, Department of Mechanical Engineering, University of California-Santa Barbara, Engineering II, Santa Barbara, CA 931065070

Dual phase titanium alloys, such as Ti-6242, experience a significant reduction in fatigue lifetime when the peak load is held at each cycle. This type of sustained peak loading, also known as dwell fatigue, mimics the long periods of high mean stress experienced by titanium fan and compressor components during takeoff and cruise. The reduction in fatigue lifetime is known as the dwell debit, and is attributed to the phenomenon of load shedding. Both local microstructure and temperature are known to impact load shedding and thereby the macroscopic response of Ti-6242 when subject to dwell fatigue, but the underlying mechanisms are still under active investigation. This study utilized electron backscatter diffraction (EBSD) and digital image correlation (DIC) to characterize the role of local microstructure and temperature on load shedding during dwell fatigue. EBSD was used to determine local orientation and texture information, and DIC provided information about the heterogeneity of the strain distribution and plastic strain accumulation. Ex-situ tests were performed to investigate the link between the deformation of local microstructures and macroscopic damage. The resultant strain fields and orientation maps were statistically analyzed to provide quantitative insights into the impact of local microstructure on load shedding during dwell fatigue.

\section{$\underline{\text { I. Introduction }}$}

Near-alpha titanium alloys are utilized extensively in the aerospace industry for their high strength-to-weight ratio, tailorable mechanical properties, and corrosion resistance. Specifically, Ti-6242 is used for disks in the compressor section of gas turbine engines. Ti-6242 is used for its low cycle fatigue and creep resistance, yet one loading condition that make lifing and designing these life critical parts challenging is dwell fatigue. [1] Dwell fatigue fundamentally deviates from low cycle fatigue, in that at the peak of each loading cycle the maximum load is held. This hold a peak load mimics the long period of high mean stress that the titanium components experience during takeoff and, to a lesser extent, cruise. A significant reduction in lifetime occurs when comparing dwell fatigue lifetimes to predicted low cycle fatigue lifetimes. This reduction in lifetime can be up to 10 to 20 times for Ti- 6242 and is attributed to the phenomenon of load shedding.

Load shedding is due to the elastic and plastic anisotropy of the hep structure of the alpha grains and variation in rate sensitivity of the different families of slip systems. Alpha grains well oriented for basal and prismatic slip relative to loading are termed "soft grains," while grains that are well-oriented for pyramidal slip are termed "hard grains." In Ti-6242, deformation in hard grains is at least 3 times more difficult to activate than slip in soft grains. [2] The worst-case scenario is when a hard grain is directly adjacent to a hard grain, called a rogue pair. Upon loading below the bulk yield strength, slip occurs in the soft grain until it meets the boundary of the hard grain where is abruptly stops. [3,4] This causes the soft grain to lose its ability to carry elastic load, thereby forcing the hard grain to carry a higher elastic load and creating a region of 
elevated stress. This allows for early crack nucleation and faceting, which significantly limits the lifetime of the component. [5] The redistribution of load is known to be affected by both local microstructure and temperature, but the exact mechanisms are still under active investigation . [6,7]

Research shows that the most detrimental temperature for dwell fatigue in Ti- 6242 alloys is approximately $65^{\circ} \mathrm{C}$, and that the dwell sensitivity essentially disappears at temperatures above $200^{\circ} \mathrm{C}$, though this may be alloy and microstructure dependent. [6,8] It has been suggested that the temperature depended is due to the decrease in slip resistance between the hard and the soft grains as the temperature increases. [9] With increasing temperature, the CRSS required for pyramidal slip decreases approach to the CRSS required for basal and prismatic slip. [10] The strength mismatch between the hard and soft grains diminishes, the stress is redistributed, and the hard grain boundary no longer acts as a pile up location. A different model attributes the primary cause of the temperature dependence is due to a thermally activated dislocation escape. During material loading, dislocations move through the material until they meet an obstacle that pins them in place. At elevated temperatures, the dislocations escape the obstacle via a thermally activated escape mechanism and continue moving through the material. At $60^{\circ} \mathrm{C}$, the dwell time and obstacle escape time are comparable, which allows additional dislocations to nucleate during the dwell and for a significant pile up to occur. At $200^{\circ} \mathrm{C}$, no load shedding occurs because the time required for dislocation escape is so short that the material reaches an equilibrium before the dwell. [6] For both thermally dependent models, it is further hypothesized that the thermal reduction of the dwell debit will results in more homogeneous deformation at the meso-scale, but this has yet to be thoroughly validated. [10]

The effects of local microstructure on dwell fatigue is a complex parameter that still requires further research. The specific microstructural features of interest relative to load shedding are microtextured regions (MTRs). MTRs are defined as clusters of similarly oriented alpha grains and can be described by their intensity (ie size, area fraction, density, and angular spread). MTRs is known to assist in the formation of fatigue cracks on both the global and local level. Locally, soft MTRs have an increased potential for closely packed hard-soft alpha particles which allow for numerous crack initiation sites that can coalesce to create a large crack growth event. [11] On the global scale, clusters of soft grains can allow for easy slip transmission between grains of nearly identical orientation, which can create slip lengths and dislocation pile ups many times larger than an individual grain. $[11,12,13,14]$

These mechanistic hypotheses have yet to be directly observed or rigorously tested. The present study investigates the mechanisms behind the temperature and microstructural dependence of load shedding to inform modeling and to improve dwell fatigue resistance and lifing of Ti-6242. Electron Backscatter Diffraction (EBSD) and Digital Image Correlation (DIC) are combined to experimentally quantify the global and local damage relative to microstructure during dwell fatigue loading. DIC is a high-resolution deformation tracking methodology that tracks a random speckle pattern from image to image. Local microstructure information collected from EBSD is spatially aligned with plastic strain maps from DIC. The resulting aligned microstructural and damage maps are analyzed to determine the microstructural impact on load shedding and the dwell debit. Quantitative mean field and local strain information over several grains and textured regions provide statistically relevant data to identify critical factors impacting the dwell debit. The slip activity and the distribution of plastic strain within MTRs and grains elucidate how dislocations move and pile up during dwell loading. This information serves to validate and further inform the mechanistic understanding of load shedding.

\section{Material and Experiments}

Dog bones were machined via wire EDM from forged and hot-rolled Ti-6242. The materials was previously beta annealed and hot rolled to a $75 \%$ reduction. The material was specifically processed to produce large MTRs on the order of $1-3 \mathrm{~mm}$. The samples were metallographically prepared via polishing down to a mirror finish with a 1:4 hydrogen peroxide: colloidal silica mixture. The resulting surfaces were marked with platinum markers via vapor deposition to define a region of interest of $4 \mathrm{~mm} \times 2.5 \mathrm{~mm}$ in the center of the gauge. The microstructure of the entire RIO was inspected using the EBSD, as shown in Figure 1. EBSD was performed with a step size of $2 \mu \mathrm{m}$ over the $4 \mathrm{~mm}$ by $2.5 \mathrm{~mm}$ field of view to 
collect information about the alpha and beta phase of the material. The material has an equixed micrstructure with an average alpha nodule size of $10 \mu \mathrm{m}$.

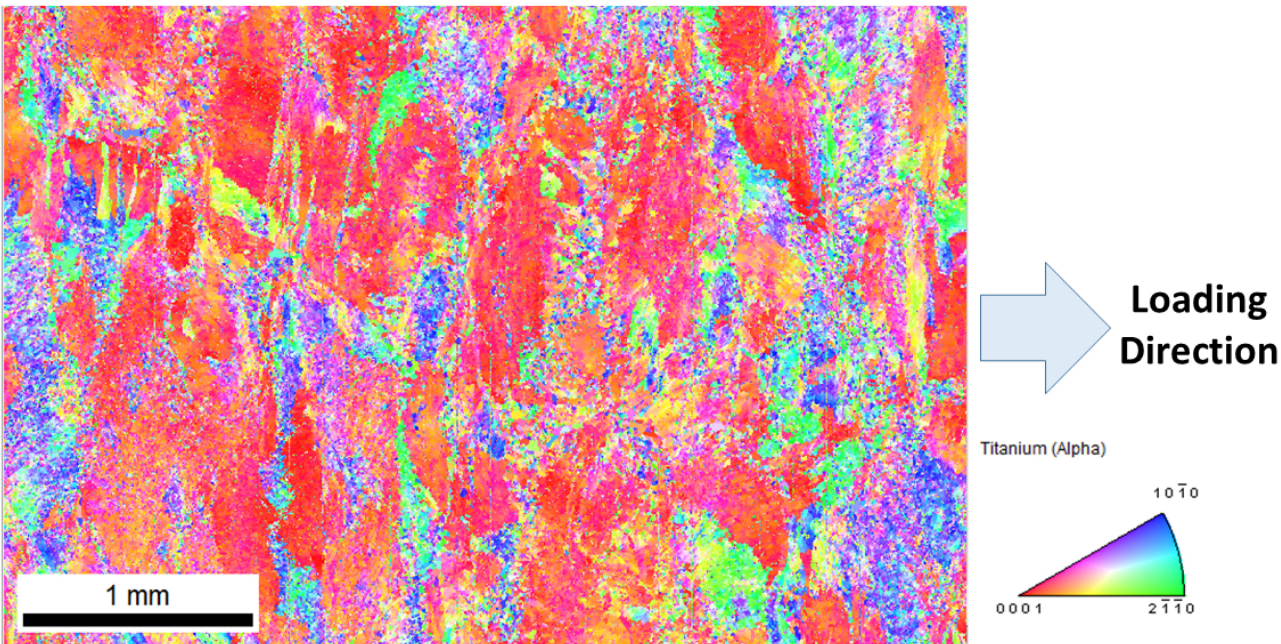

Figure 1: EBSD IPF Map Relative to Loading Direction

$300 \mathrm{~nm}$ gold nanoparticles were then attached following [15] to act as a speckle pattern for SEM DIC. The sample was then loaded via dwell fatigue to $90 \%$ of the macroscopic, room temperature yield ( $905 \mathrm{MPa}$ ) of the material for 200 cycles. Each cycle was comprised of a 1 second load to $814.5 \mathrm{MPa}$, a 120 hold a peak load, and then 1 second unload in load control with an R ratio of zero. Loading was interrupted at various cycles (after cycle 1, 2, 5, 50, 100, 200) and the sample was imaged in-SEM for DIC analysis. 198 individual tiles were collected with an external scan controller, correlated with VIC-2D 6 (Correlated Solutions), and stitched together to create a continuous plastic strain map over the entire region of interest made up of 250 million data points per strain step, as seen in Figure 2. [16,17] The platinum markers were used to align the microstructural map and the strain map via projective transformations. 


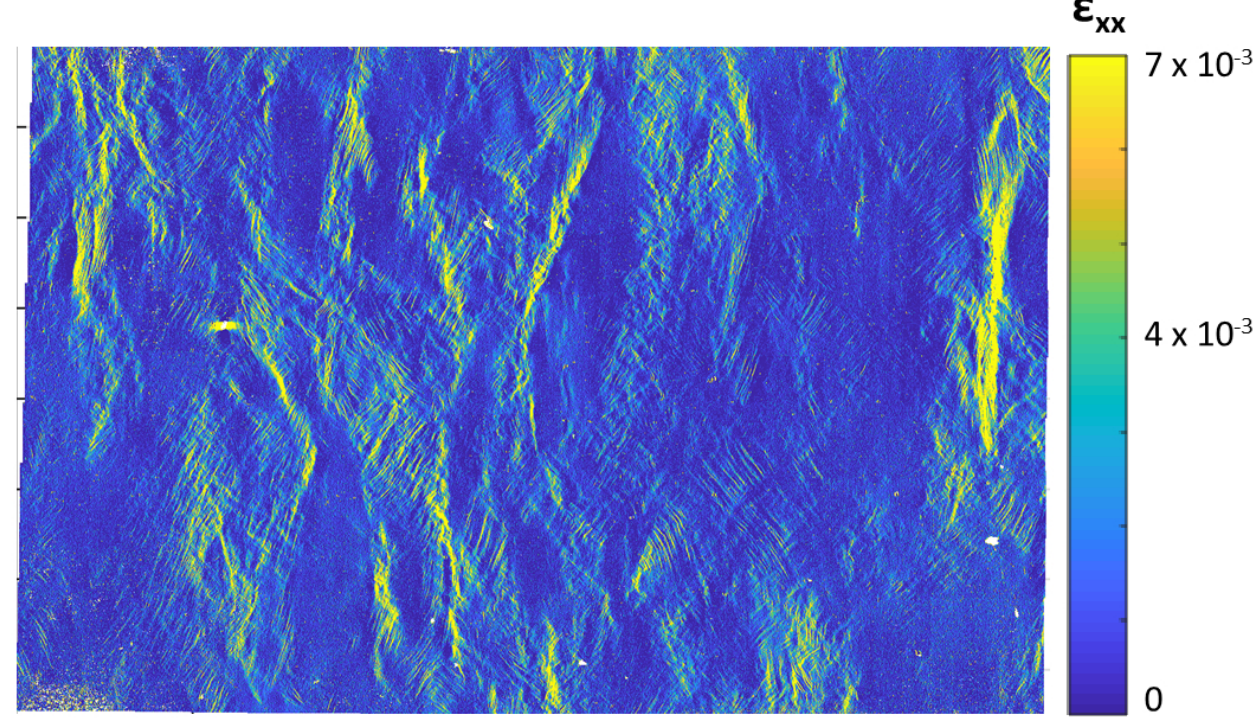

Figure 2: Plastic Strain Accumulation After 200 Cycles

The individual slip and pile up events were segmented from the strain map using a process of data clustering, image processing, and Hough transforms, show in Figure 3. [18] The active slip type of many long-range slip features were identified by comparing the theoretical slip trace of the grains involved in the long-range slip event and the observed identified Hough trace from the slip in the strain map. [19,20,21] This process was performed in a semi-automated fashion and checked by hand. Only slip traces within two degrees of the predicted slip trace are considered identifiable. Otherwise, the slip trace was not included in the analysis of active slip type. The results and implications of these segmented strain and microstructural maps for cycle 2 and 200 are in the following Results and Discussion. 


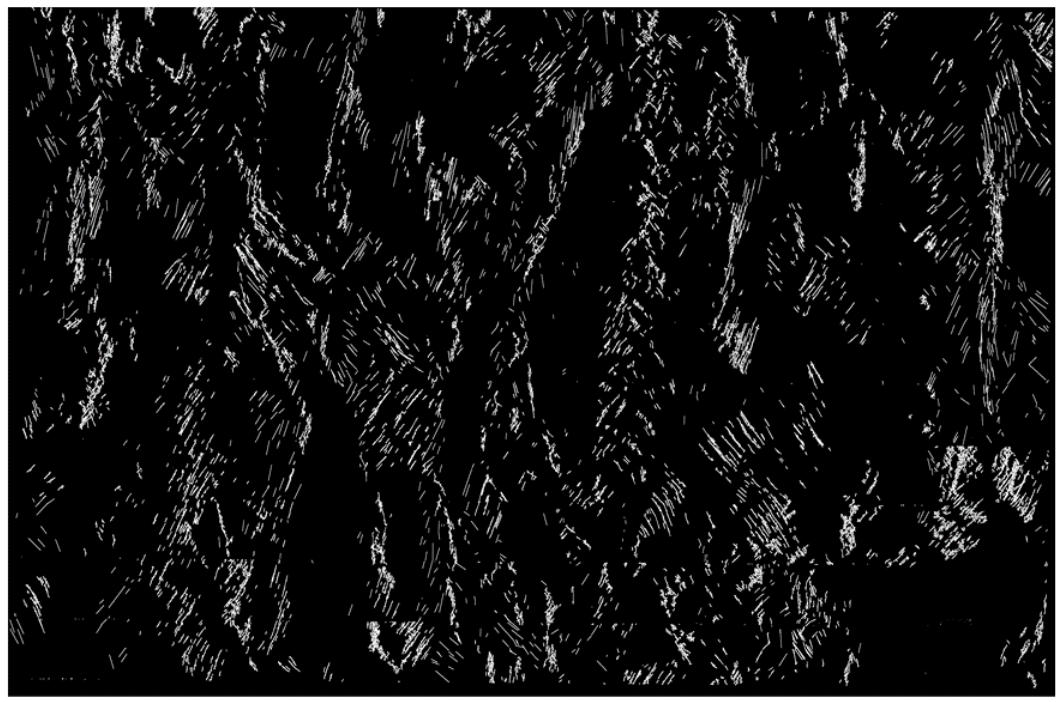

Figure 3: Binary Map of Segmented Strain Features After 200 Cycles

\section{Results and Discussion}

By aligning the orientation information collected with EBSD with the strain maps from SEM DIC, the long-range slip traces can be matched to the orientation of the individual grains involved in the cooperative slip transfer. Long-range slip in this study is defined as slip traces that extend beyond two grains. By extracting all the orientation information from a segmented slip trace, the dominant orientation of the grains involved in that slip trace can be found by determining the statistical mode of all the orientations. Each trace's dominant orientation can be used to calculate the Schmid factor for that longrange slip trace. The dominant orientation of all detected slip traces had a high basal Schmid factor, as shown Figure 4. Cooperative slip can occur through multiple grains where they individually exhibit a high basal Schmid factors and are co-located. 


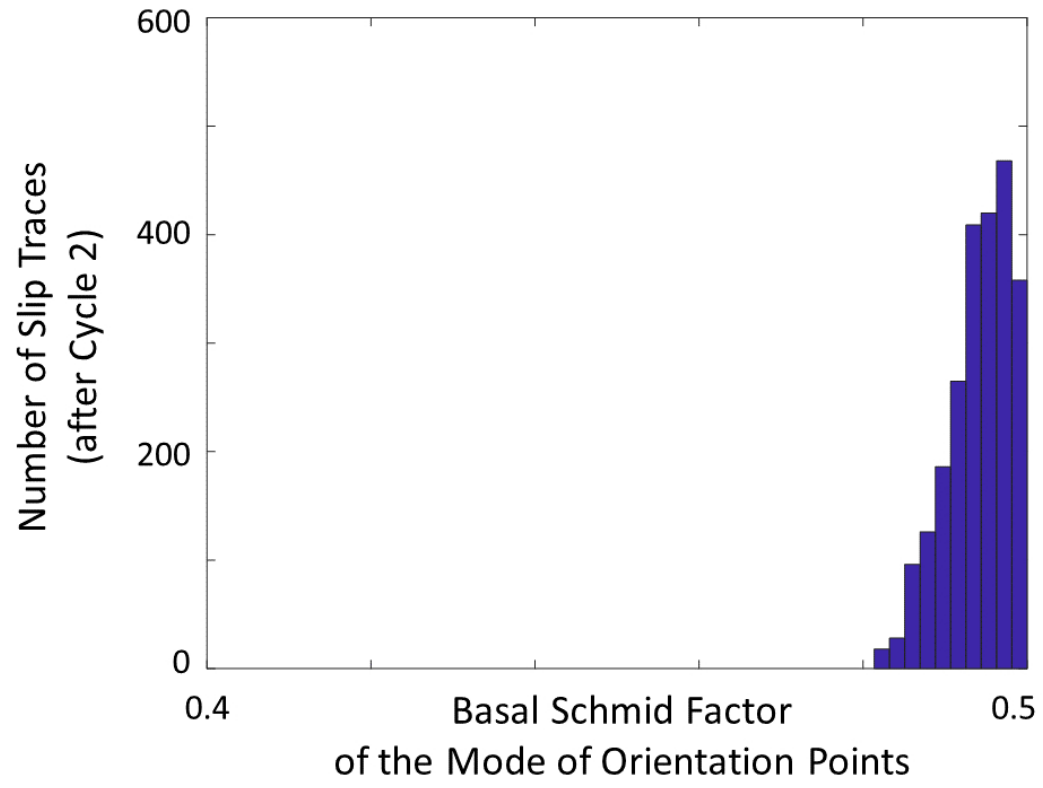

Figure 4: Basal Schmid Factor for the Mode Orientation of Each Segmented Slip Trace After 2 Dwell Cycles

Additionally, the distribution of orientations for each long-range slip trace is small as seen in Figure 5 . Long-range slip features occur through groups of grains with a similar ease of basal slip. The distribution of grain orientations an individual slip feature extends through is small.

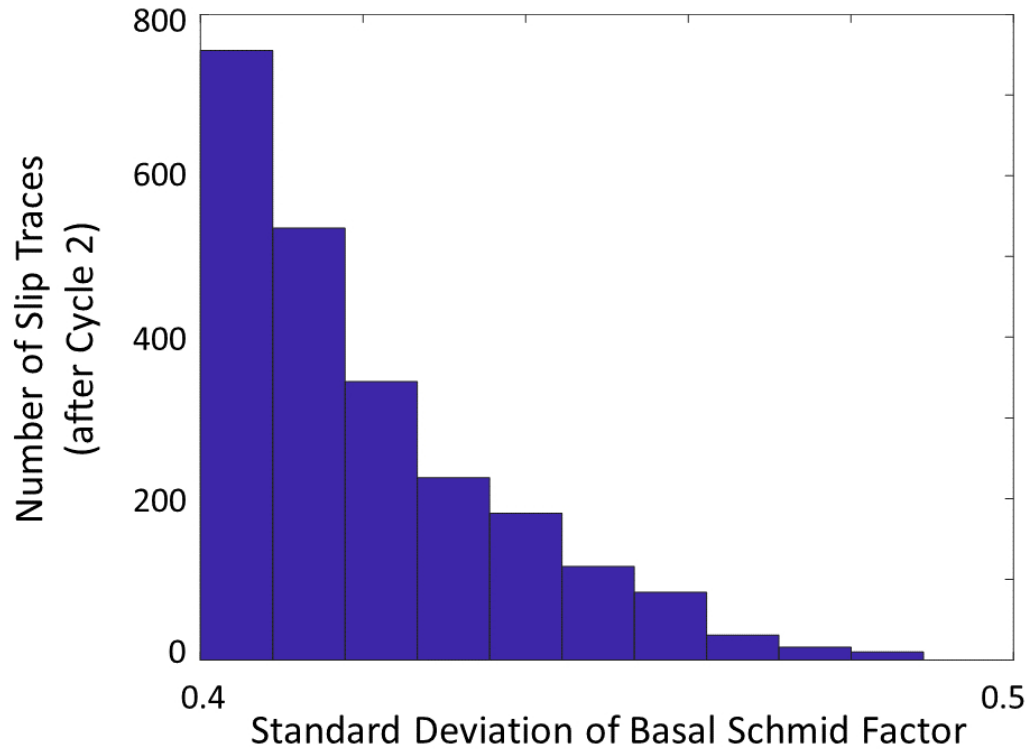


Figure 5: Standard Deviation of Basal Schmid Factor Per Slip Trace After 2 Dwell Cycles

Identification of the active slip systems of many of the long-range slip traces reveals that plastic deformation is predominantly accommodated by basal slip with very little long-range prismatic slip Figure 6. Even in MTRs that exhibit a high pristmatic schmid factor, little to no prismatic slip is observed. This trend is exhibited after only two cycles. Long-range pyramidal slip is also identified. This slip is hypothesized to exist to allow additional deformation in regions that have already deformed by basal slip as well as to maintain compatibility at the interface between deformed and undeformed regions. More rigorous inspection and further testing is required to determine the cause of this behavior. With increased cycling, the total number of slip features increases and the relative ratios of identified basal, prismatic and pyramidal remain consistent. Basal slip dominates the identified deformation at both early and late cycles.

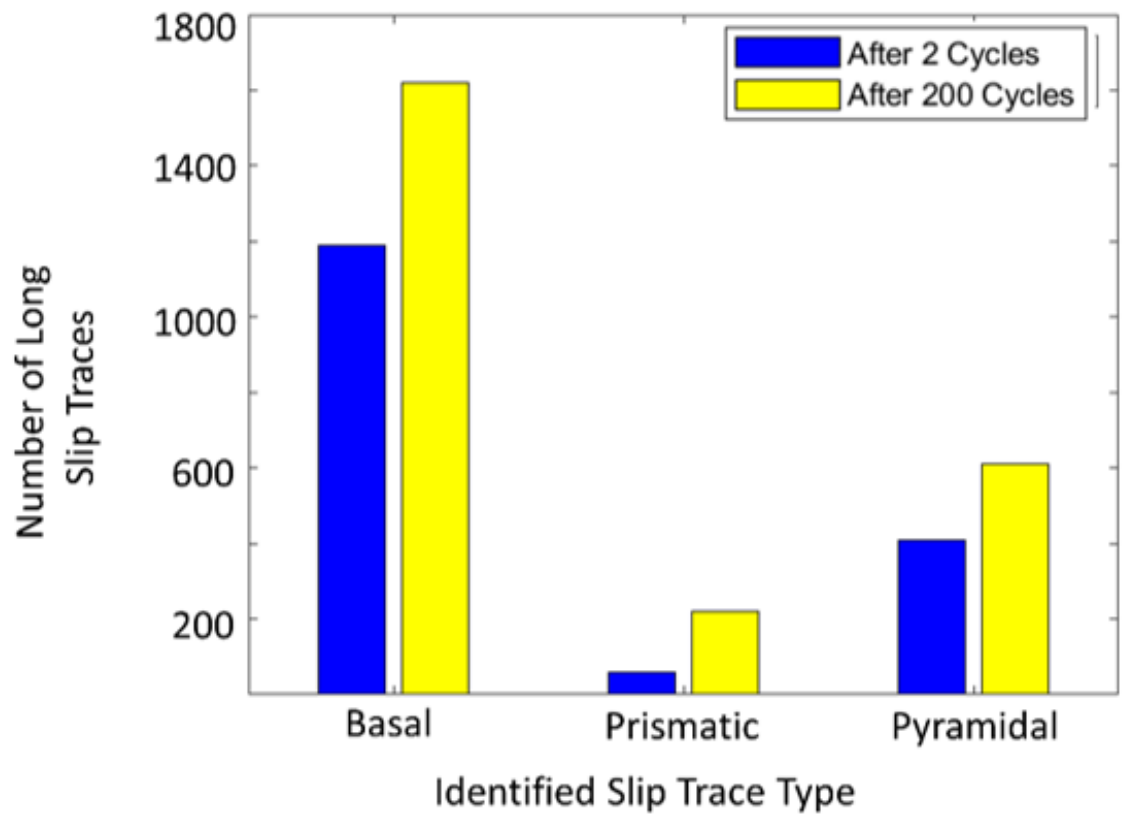

Figure 6: Active Slip System for the Identified Slip Traces After 2 and 200 Dwell Cycles

This contrasts with the predominant active slip identified in individual grains and MTRs in titanium alloys during tensile and dwell fatigue loading by various independent groups. [12,21,22,23] Echlin et al identified mostly prismatic slip band across the MTRs of inspection under in-situ tensile loading of Ti-6-4 below the bulk yield strength. [12] Zhang et al (quant) experimentally observed both basal and prismatic slip activity after loading to $\sim 3 \%$ strain in Ti-6-4, but that prismatic slip dominated. [23] The same experiment by Zhang et al (quant) and a dwell fatigue experiment on Ti-6242 by Hemery(comparison) observed that most of the deformation was accommodated by individual soft grains with a Schmid factor above 0.4 , but in both cases there was a non-negligible amount of slip occurring in grains with a Schmid factor of 0.2 to 0.4. [21,23] Every longrange slip trace from the present study has a basal Schmid factor above 0.4. It is interesting to note that Ti-6-4 only exhibits a dwell debit of about 2 to 3 times the LCF lifetime while Ti-6242 has a dwell debit of 10-20 times the LCF lifetime at room temperature when loaded to $90 \%$ of the macroscopic yield strength. [4] The observed active slip systems and Schmid factors in this study may speak to the requirements for slip transfer between soft grains as well as indicate how the behavior of MTRs differs from the behavior of very large grains. More analysis and testing are 
required to further elucidate the trends and mechanisms that allow for cooperative slip in Ti-6242 and its implications on load shedding and dwell fatigue.

\section{Conclusions}

This ex-situ study of plastic strain accumulation in Ti-6242 under dwell fatigue loading inform our understanding of MTRs and their effects on dwell fatigue lifetimes. At room temperature, plastic strain accumulation is observed at MTR interfaces at the microscale. Also, contiguous slip occurs across continuous clusters of grains. These long-range slip features are dominantly basal slip and occur through grains with a high basal Schmid factor and a small spread in orientation. Further segmentation and statistical analysis of this data set and additional dwell fatigue experiments, specifically at elevated temperature, are needed to further elucidate the role of microstructure and temperate on load shedding.

\section{$\underline{\text { V. Acknowledgments }}$}

This material is based upon work supported by the Air Force Research Labs and the National Science Foundation Graduate Research Fellowship under Grant No. 1256260 DGE. We would also like to acknowledge our collaborators, Mr. Kirk Fields at UCSB and my fellow Daly Research Group members.

\section{References}

1. Cuddihy, M. A., Stapleton, A., Williams, S., Dunne, F. P. E. (2016). International Journal of Fatigue, 97, 177-189.

2. Gong, J., Wilkinson, A.J. (2019). Acta Materialia, 57(19) 5693-5705.

3. Evans, W.J., Bache M.R. (1994). International Journal of Fatigue, 16 (7), $43-52$.

4. Bache MR. (2003). International Journal of Fatigue, 25, 1079-1087.

5. Hasija, V., Ghosh, S., Mills, M.J., Joseph, D.S. (2003). Acta Materialia, 51, 4533-4549.

6. Zheng,Z., Balint, D., Dunne,F.P.E. (2017). Journal of the Mechanics and Physics of Solids, 107, 185-203.

7. Zhang, Z., Cuddihy, M.A., Dunne, F.P. (2015). Proceedings Royal Society A, 471: 20150214.

8. Winstone M.R., Weaver M.J. (1984). Titanium science and technology: proceedings of the fifth International Conference on Titanium, vol. 4.

9. Ozturk, D., Shahba, A., Ghosh, S. (2016). Fatigue Fracture Engineering Material Structures, 39, 752-769.

10. Williams, J.C., Baggerly, R.G., Paton, N.E. (2002). Metallurgical and Materials Transactions A, 33(3), 837-850.

11. Bantounas, I., Lindley, T. C., Rugg, D., Dye, D. (2007). Acta Materialia, 55, 5655-5665.

12. Echlin, M.P., Stinville, J.C., Miller, V.M., Lenthe, W.C., Pollock, T.M. (2016). Acta Materialia, 114,164-175.

13. Venkatramani, G., Ghosh, S., \& Mills, M. (2007). Acta Materialia, 55(11), 3971-3986.

14. Lunt, D., da Fonseca, J. Q., Rugg, D., \& Preuss, M. (2017). Materials Science and Engineering A, 680, 444-453.

15. Kammers, A. D., \& Daly, S. (2011). Measurement Science and Technology, 22(12), 125501.

16. Lenthe,W., Stinville, J.C., Echlin, M., Chen, Z., Daly, S. Pollock, T. (2018). Ultramicroscopy, 195, 93-100.

17. Chen, Z., Lenthe, W., Stinville, J.C., Echlin, M., Pollock, T.M., Daly, S. (2018). Experimental Mechanics, 58(9), 1407-1421.

18. Chen, Z., Daly, S. (2018). Materials Science and Engineering A, 736, 61-75.

19. Chen, Z., Daly, S. H. (2017). Experimental Mechanics, 57(1), 115-127.

20. Bridier, F., Villechaise, P, Mendez, J. (2005). Acta Materialia, 53, 555-567.

21. Hémery, S., Villechaise, P. (2017). Materials Science and Engineering A, 697(March) 177-183.

22. Hémery, S., Dang, V. T., Signor, L., Villechaise, P. (2018). Metallurgical and Materials Transactions A, 49(6), 2048-2056. 
23. Zhang, Z., Lunt, D., Abdolvand, H., Wilkinson, A. J., Preuss, M., Dunne, F. P. E. (2018). International Journal of Plasticity, 108(April), 88-106. 